\title{
Using Matlab-generated Numerical Solutions in an Environmental Engineer- ing Class to Predict the Fate and Transport of Contaminants
}

Dr. Alexa Rihana-Abdallah, University of Detroit Mercy

Alexa Rihana-Abdallah is a professor of civil and environmental engineering at the University of Detroit Mercy,rihanaa@udmercy.edu

\section{Dr. James Joseph Lynch, University of Detroit Mercy}

Dr. Lynch is an Associate Professor in the Department of Civil, Architectural, and Environmental Engineering. He teaches classes in geotechnical engineering, construction materials, and forensic engineering. His research interests include nondestructive testing based on wave propagation methods. He is a licensed professional engineer in the State of Michigan. He is the advisor for the student chapter of the American Society of Civil Engineers and the concrete canoe team. 


\title{
Using Matlab-generated Numerical Solutions in an Environmental Engineering Class to Predict the Fate and Transport of Contaminants
}

\begin{abstract}
Contaminant transport and fate is an important topic in environmental engineering. Closed-form solutions exist for homogeneous domains; however actual contaminated sites are rarely homogeneous, which means that no-closed form solution exists. An alternative to closedform solutions is a numerical solution through computer programs. Although commerciallyavailable software packages have been written to model contaminant transport and fate, a student-written program is chosen in this work for pedagogical reasons. With contaminant transport being an application of the differential equations, students in the senior-level environmental engineering class, Hazardous Waste, were introduced to analytical and numerical solutions of the partial differential equations governing contaminant transport, and then they used Matlab to investigate and predict the contaminant behavior in time and space, by modeling the data available from the Environment Protection Agency (EPA) website of a contaminated site. Students worked in groups, for four weeks, to model the contaminant concentrations over time and distance. A survey at the end of the project and comments from the course evaluations indicate that the Matlab modeling exercise, although challenging, was well received. Students were able to predict the time when the concentration of the contaminant drops below the maximum allowable concentration in drinking water. They also were able to model the movement of the plume. When asked about the importance of learning a computer language, 92\% stated that this skill and knowledge are very important and will help them in their job
\end{abstract}


applications, $76 \%$ stated that modeling the plume helped them visualize its spread and predict its fate. For future offerings, more time will be provided for the project and time will be allocated in class to work on the project. In addition, sites with time-varying boundary conditions will be introduced, as well as student assessment prior and after completing the computer assignment.

\section{Introduction}

The civil and environmental engineering program at our university is broad-based. In addition to classes in math, physical sciences, engineering analysis, and engineering design, all students take one computer programming class, $\mathrm{C}++$, in their sophomore year. This class develops logical thinking through algorithm design and flowcharts. Recognizing the importance of structured programming and building on the acquired knowledge in the $\mathrm{C}++$ course, this paper presents the results of a computer project given in an undergraduate environmental engineering class, CIVE 4830 - Hazardous Waste. The class is a senior level class, taken by students pursuing an environmental concentration or planning on continuing their graduate studies in environmental engineering. This class covers topics that include identifications and classifications of hazardous waste, physical and chemical properties of pollutants, toxicity assessment, fate and transport of contaminants, and remediation techniques. Introducing a computer modeling project was a natural addition to the fate and transport section of the course, and supplemented well the concepts presented there.

The use of computers has become a necessary part of the education of students, as well as the practice of engineering. Students have traditionally used Microsoft Excel in engineering classes to perform repetitive calculations such as analyzing laboratory data and solving 
homework problems. While Microsoft Excel works well for some applications, it was not designed for the applications that frequently appear in upper-level engineering classes, such as the solution of differential equations. Computer applications provide the technical benefits of quickly solving a large number of problems, but can also provide educational benefits, if properly implemented. Students can improve their programming skills, which can be an important skill for practicing engineers (Dunn et al., 2005), and can learn topics such as model validation and sensitivity (Maase, 2007).

The project presented in this work involves the modeling of a contaminant transport and fate. Many commercially-available software packages have been written to model contaminant transport and fate; however, a student-written Matlab ${ }^{\circledR}$ program was selected primarily for pedagogical reasons. It allows students to improve their programming skills, and students generally have a positive response to these assignments (Cretchley et al., 1999) without the fear of turning an engineering class into a computer programming class. Working in groups of four, for four weeks, students used MATLAB to simulate changes in the concentration of a contaminant, trichloroethylene (TCE), over time and distance. They created a computer program that would display models of the contaminant concentration vs. time and its concentration vs. distance. The data used are taken from the EPA 2010 Annual Status Report for the Boomsnub/Airco Superfund Site, in Hazel Dell, Washington.

\section{Contaminant Transport and Fate}

In this section, students were first introduced to the comparison of numerical and analytical solutions to the governing equations of contaminant transport. Soil and groundwater 
systems can become contaminated when, for example, a tanker truck is involved in an accident, when an underground storage tank breaks, or when an industrial facility spills chemicals. The contaminant flows out through the unsaturated soil and reaches the water table. Once the contaminant reaches the water table, the contaminant could stay atop the water table if it is a light non-aqueous phase liquid (L-NAPL), it could pass through the saturated zone until it reaches an impervious layer if it is a dense non-aqueous phase liquid (D-NAPL), or it could dissolve into the water. The movement and fate of the contaminant once it reaches the saturated zone depends on the soil-groundwater system.

The concentration of the contaminant in the plume will vary with time and space. Modeling the contaminant transport and fate requires the identification of the processes that affect the movement of the contaminant. Advection is an important process that drives the contaminant to migrate downgradient the saturated zone. It is the transport process of chemicals along the groundwater paths without causing any reduction in the contaminant concentration or mass. Another process is the hydrodynamic dispersion which encompasses molecular diffusion and mechanical dispersion. This mixing process spreads the contaminant plume out of the groundwater flow direction. In most cases, mechanical dispersion dominates the process since molecular diffusion is extremely slow. Microbial or chemical reactions are also processes that play a role in contaminant transport. Developing and solving the corresponding governing equations depend on whether advection, dispersion, and reaction are present. 
For simple domains, i.e., homogeneous properties and simple boundary conditions, the governing equation can be solved analytically. In general, an analytical solution does not exist and the governing equation must be solved numerically.

\section{Contaminant Transport by Advection-Dispersion}

A model that includes only advection and dispersion can be solved numerically and may be solved analytically for certain domains and properties. The contaminant will flow downstream with the groundwater and the front will spread out through dispersion. The governing equation is

$$
\frac{\partial C(x, t)}{\partial t}=D \frac{\partial^{2} C(x, t)}{\partial x^{2}}-v \frac{\partial C(x, t)}{\partial x}
$$

where $C$ is the concentration, $D$ is the dispersion coefficient, and $v$ is the groundwater velocity. The domain for the problems considered herein is defined by

$$
x>0, \quad t>0
$$

where the initial condition is

$$
C(x, 0)=0, \quad x \geq 0
$$

and the boundary conditions are

$$
\begin{gathered}
C(0, t)=C_{0}, \quad t \geq 0 \\
|C(\infty, t)| \leq \infty, \quad t \geq 0
\end{gathered}
$$

The second part of equation 4 requires that the function exists as $x$ approaches infinity.

\section{Analytical Solution}

Equation 1 subject to the conditions of equations 2 through 4 has been solved analytically (Ogata \& Banks, 1961), and solution to this equation is 


$$
\frac{C(x, t)}{C_{0}}=\frac{1}{2}\left[\operatorname{erfc}\left(\frac{x-v t}{\sqrt{4 D t}}\right)+\exp \left(\frac{x v}{D}\right) \operatorname{erfc}\left(\frac{x+v t}{\sqrt{4 D t}}\right)\right]
$$

where $\operatorname{erfc}$ is the complementary error function.

\section{Numerical Solution}

The finite difference method is one method of numerically solving partial differential equations (Burden and Faires, 2001). In this method, the domain is divided into a grid, derivatives are approximated by the difference in the value of the function divided by the grid size, and the values of the function are computed by solving a system of equations. The error in the numerical solution is controlled by the expression of the derivative, the grid size for approximation in the spatial derivative, and the step size in the approximation of the time derivative.

The differential equation defined in equation 1 can be formulated by the finite difference method to second-order accuracy in both time and space by the Crank-Nicholson method (Burden and Faires, 2001). In the Crank-Nicholson method, the derivatives are approximated for the current time step and the next time step using the values of the function at the grid points. All values at the current time step are known, and the values at the next time step are computed by solving a system of algebraic equations.

The domain is divided into a grid with $M$ points and is solved over $N$ time steps. The grid size is $\Delta \mathrm{x}$, and the time step size $\Delta \mathrm{t}$. 
The approximation for the first derivative of the concentration with respect to $x$ at an arbitrary point, $m$, at the current time step, $n$, is

$$
\frac{\partial C_{m, n}}{\partial x} \approx \frac{C_{m+1, n+1}-C_{m-1, n}}{2 \Delta x}
$$

The approximation for the second derivative of the concentration with respect to $x$ at an arbitrary point, $m$, at the current time step, $n$, is

$$
\frac{\partial^{2} C_{m, n}}{\partial x^{2}} \approx \frac{C_{m+1, n}-2 C_{m, n}+C_{m-1, n}}{\Delta x^{2}}
$$

The approximations of the spatial derivatives at the next time step, $n+1$, are defined by replacing the subscript $n$ by $n+1$ in equations 6 and 7 .

The approximation for the first derivative of the concentration with respect to $t$ at an arbitrary point, $m$, at the current time step, $n$, is

$$
\frac{\partial C_{m+1, n}}{\partial t} \approx \frac{C_{m, n+1}-C_{m, n}}{\Delta t}
$$

Combining the terms in equation 1 with the average of the spatial derivatives at the current and next time step produces

$$
\frac{C_{m, n+1}-C_{m, n}}{\Delta t}=\left(\frac{1}{2}\right)\left[\begin{array}{l}
\left(\begin{array}{l}
D \frac{C_{m+1, n+1}-2 C_{m, n+1}+C_{m-1, n+1}}{\Delta x^{2}} \\
-v \frac{C_{m+1, n+1}-C_{m-1, n+1}}{2 \Delta x}
\end{array}\right) \\
+\left(\begin{array}{l}
D \frac{C_{m+1, n}-2 C_{m, n}+C_{m-1, n}}{\Delta x^{2}} \\
-v \frac{C_{m+1, n}-C_{m-1, n}}{2 \Delta x}
\end{array}\right)
\end{array}\right]
$$


Rearranging the terms in equation 9 produces

$$
2\left(C_{m, n+1}-C_{m, n}\right)=\left[\begin{array}{l}
\left(\begin{array}{l}
\frac{D \Delta t}{\Delta x^{2}}\left(C_{m+1, n+1}-2 C_{m, n+1}+C_{m-1, n+1}\right) \\
-\frac{v \Delta t}{2 \Delta x}\left(C_{m+1, n+1}-C_{m-1, n+1}\right)
\end{array}\right) \\
+\left(\begin{array}{l}
\frac{D \Delta t}{\Delta x^{2}}\left(C_{m+1, n}-2 C_{m, n}+C_{m-1, n}\right) \\
-\frac{v \Delta t}{2 \Delta x}\left(C_{m+1, n}-C_{m-1, n}\right)
\end{array}\right)
\end{array}\right]
$$

For simplification, the constants on the right-hand side of equation 10 can be replaced by

$$
\begin{aligned}
& \lambda=\frac{D \Delta t}{\Delta x^{2}} \\
& \mu=-\frac{v \Delta t}{2 \Delta x}
\end{aligned}
$$

Substituting the constants defined by equations 10 and 11 into equation 12 and rearranging so that all of the unknown quantities are on the left-hand side and all of the known quantities are on the right-hand side produces

$$
\begin{aligned}
& -(\lambda-\mu) C_{m-1, n+1}+2(1+\lambda) C_{m, n+1}-(\lambda+\mu) C_{m+1, n+1} \\
& =(\lambda-\mu) C_{m-1, n}+2(1-\lambda) C_{m, n}+(\lambda+\mu) C_{m+1, n}
\end{aligned}
$$

which is the discrete form of equation 1 for one grid point. Once the domain is divided into $\mathrm{M}$ points, equation 13 holds true for the interior grid points, but some modifications are required for the first grid point and the last grid point. At the first grid point, $m$ is equal to 1 , and $\mathrm{C}_{\mathrm{m}-1, \mathrm{n}+1}$ and $\mathrm{C}_{\mathrm{m}-1, \mathrm{n}}$ are at the left boundary. The value of $\mathrm{C}$ at the left boundary is $\mathrm{C}_{0}$ for all time steps, from which equation 13 becomes 


$$
\begin{aligned}
2(1+\lambda) C_{1, n+1}-(\lambda+\mu) C_{2, n+1} & =2(1-\lambda) C_{1, n}-(\lambda+\mu) C_{2, n} \\
& +2(\lambda+\mu) C_{0}
\end{aligned}
$$

and at the last grid point, $m$ is equal to $M$, which is the number of grid points, $C_{m+1, n+1}$ and $C_{m+1, n}$ are at the right boundary. The value of $\mathrm{C}$ at the right boundary is 0 for all time steps, from which equation 13 becomes

$$
-(\lambda-\mu) C_{M-1, n+1}+2(1+\lambda) C_{M, n+1}=(\lambda-\mu) C_{M-1, n}+2(1-\lambda) C_{M, n}
$$

Equations 11 through 15 have been coded by the instructor into a Matlab ${ }^{\circledR}$ script for analysis of the contaminant transport by advection and dispersion. The code was given and explained to the students. The results of the simulation for the numerical and analytical solutions are presented in Figure 1. The code and instructions for using the code are available from the authors.

\section{Comparison of Analytical and Numerical Results}

Partial validation of the finite difference formulation of equation 1 has been performed by solving the finite difference formulation, and comparing those numerical results to the analytical solution expressed by equation 5 . The convergence of the finite difference solution to the analytical solution is measured by computing the vector of differences between the two sets of values, and dividing the $\mathrm{L}_{2}$ norm of that vector by the $\mathrm{L}_{2}$ norm of the vector of analytical values. The parameters used in the analysis are defined in Table 1. 
Table 1: Parameters used in validation of one-dimensional advection-dispersion model

\begin{tabular}{|c|c|c|}
\hline \multicolumn{2}{|c|}{ Property } & Value \\
\hline \multirow[t]{2}{*}{ Domain } & Left & $0 \mathrm{~m}$ \\
\hline & Right & $10 \mathrm{~m}$ \\
\hline \multicolumn{2}{|l|}{ Time } & 1 day \\
\hline \multirow{2}{*}{$\begin{array}{l}\text { Boundary } \\
\text { conditions }\end{array}$} & Left & 1 \\
\hline & Right & 0 \\
\hline \multicolumn{2}{|l|}{ Initial condition } & 0 \\
\hline \multirow{2}{*}{$\begin{array}{l}\text { Transport } \\
\text { properties }\end{array}$} & Velocity & $1 \mathrm{~m} /$ day \\
\hline & Dispersion & $0.5 \mathrm{~m}^{2} / \mathrm{day}$ \\
\hline \multirow[t]{2}{*}{ Discretization } & $\mathrm{dx}$ & $0.01 \mathrm{~m}$ \\
\hline & $\mathrm{dt}$ & 0.01 day \\
\hline
\end{tabular}

The results of the simulation are presented in Figure 1.

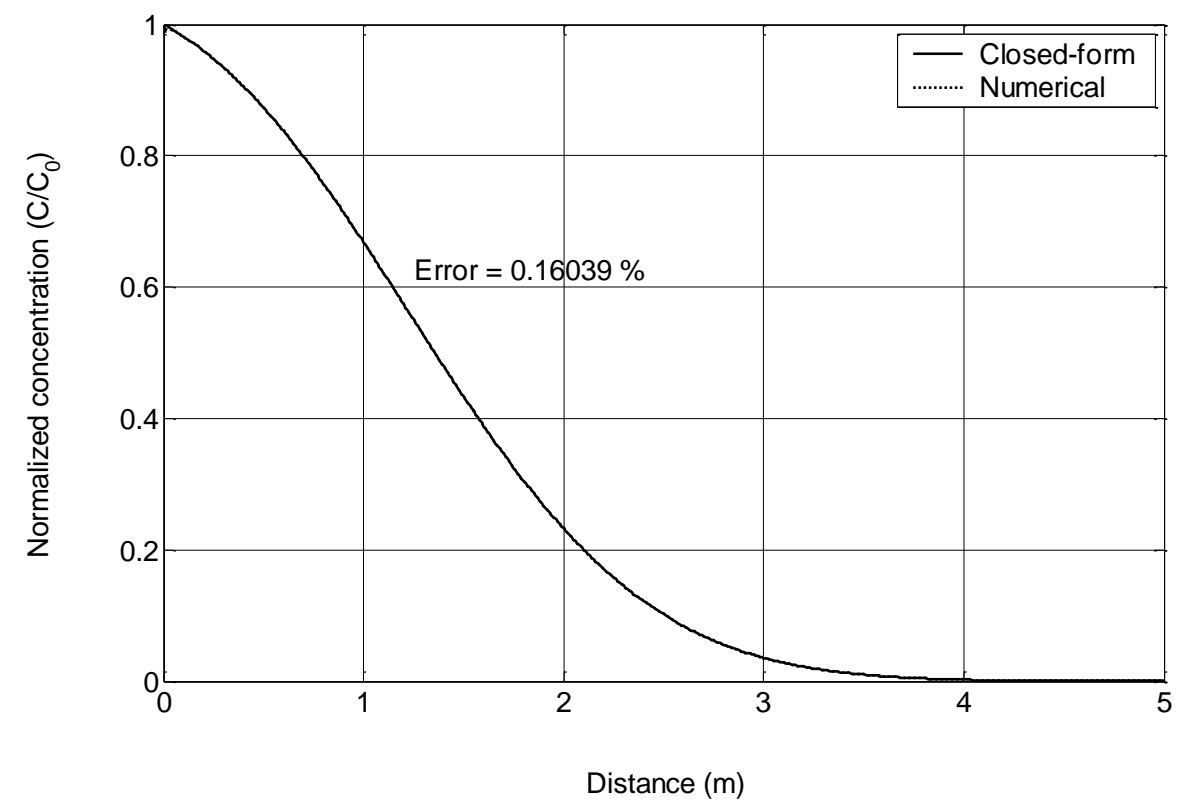

Figure 1: Advection-dispersion results, analytical and numerical solutions 
The analytical and numerical results are so close to each other that two distinct curves may be difficult or impossible to see. The error is on the order of $0.16 \%$, which is considered to be acceptable.

\section{Contaminant Transport by Advection, Dispersion, and Reaction}

Attenuation of the contaminant occurs as chemicals in the soil-groundwater system react with the contaminant or as microbes in the soil-groundwater system consume the contaminant. Many systems are modeled using first-order kinetics, in which the attenuation depends on the concentration of the contaminant. The governing differential equation for advection, dispersion, and reaction, assuming first-order kinetics for the decay, is

$$
\frac{\partial C}{\partial t}=D \frac{\partial^{2} C}{\partial x^{2}}-v \frac{\partial C}{\partial x}+\beta C
$$

Equation 16 is a modification of equation 1, with the addition of the attenuation, which is the attenuation constant, $\beta$, multiplied by the concentration, $C$.

\section{Analytical Solution}

Equation 16 subject to the conditions of equations 2 through 4 has been solved analytically (Bear, 1979), and the solution to this equation is

$$
\frac{C(x, t)}{C_{0}}=\frac{\exp \left(-k_{3} x\right)}{2}\left[\operatorname{erfc}\left(\frac{x-k_{2} t}{k_{1}}\right)+\exp \left(k_{3} x\right) \operatorname{erfc}\left(\frac{x+k_{2} t}{k_{1}}\right)\right]
$$

The terms $\mathrm{k}_{1}, \mathrm{k}_{2}$, and $\mathrm{k}_{3}$ are constants defined by

$$
\begin{aligned}
& k_{1}=\sqrt{4 D t} \\
& k_{2}=\sqrt{v^{2}+4 \beta D}
\end{aligned}
$$




$$
k_{3}=\sqrt{\left(\frac{v}{2 D}\right)^{2}+\frac{\beta}{D}}
$$

\section{Numerical Solution}

The finite difference formulation of equation 16 for an arbitrary interior node is

$$
\begin{aligned}
& -(\lambda-\mu) C_{m-1, n+1}+(2+2 \lambda+\beta) C_{m, n+1}-(\lambda+\mu) C_{m+1, n+1} \\
& =(\lambda-\mu) C_{m-1, n}+(2-2 \lambda-\beta) C_{m, n}+(\lambda+\mu) C_{m+1, n}
\end{aligned}
$$

The finite difference formulation for the first grid point is

$$
\begin{aligned}
(2+2 \lambda+\beta) C_{1, n+1}-(\lambda+\mu) C_{2, n+1} & =(2-2 \lambda-\beta) C_{1, n} \\
& -(\lambda+\mu) C_{2, n}+2(\lambda+\mu) C_{0}
\end{aligned}
$$

The finite difference formulation for the last grid point is

$$
\begin{aligned}
-(\lambda-\mu) C_{M-1, n+1}+(2+2 \lambda+\beta) C_{M, n+1} & =(\lambda-\mu) C_{M-1, n} \\
& +(2-2 \lambda-\beta) C_{M, n}
\end{aligned}
$$

Again a Matlab ${ }^{\circledR}$ script for analysis of the contaminant transport by advection, dispersion, and reaction was provided by the instructor and explained to the students. The results of the simulation for the numerical and analytical solutions are presented in Figure 2.

\section{Comparison of Analytical and Numerical Results}

Partial validation of the finite difference formulation of equation 16 has been performed by solving the finite difference formulation, and comparing those results to the analytical solution expressed by equation 17 . As with the validation of the advection-dispersion model, the convergence of the finite difference solution to the analytical solution is measured by computing the vector of differences between the two sets of values, and dividing the $\mathrm{L}_{2}$ norm of that vector 
by the $\mathrm{L}_{2}$ norm of the vector of analytical values. The parameters used in the analysis are defined in Table 2.

Table 2: Parameters used in validation of one-dimensional advection-dispersion-reaction model

\begin{tabular}{|l|c|c||}
\hline \multicolumn{2}{|c|}{ Property } & Value \\
\cline { 2 - 3 } Domain & Left & $0 \mathrm{~m}$ \\
\cline { 2 - 3 } & Right & $10 \mathrm{~m}$ \\
\hline Time & & 1 day \\
\cline { 2 - 3 } $\begin{array}{l}\text { Boundary } \\
\text { conditions }\end{array}$ & Left & 1 \\
\hline Initial condition & 0 \\
\hline \multirow{2}{*}{$\begin{array}{l}\text { Transport } \\
\text { properties }\end{array}$} & Velocity & $1 \mathrm{~m} /$ day \\
\cline { 2 - 3 } & Dispersion & $1 \mathrm{E}-6 \mathrm{~m}^{2} /$ day \\
\cline { 2 - 3 } & Reaction & $0.01 \mathrm{day}^{-1}$ \\
\hline \multirow{2}{*}{ Discretization } & $\mathrm{dx}$ & $0.1 \mathrm{~m}$ \\
\cline { 2 - 3 } & $\mathrm{dt}$ & $0.01 \mathrm{day}$ \\
\hline
\end{tabular}

The results of the simulation are presented in Figure 2.

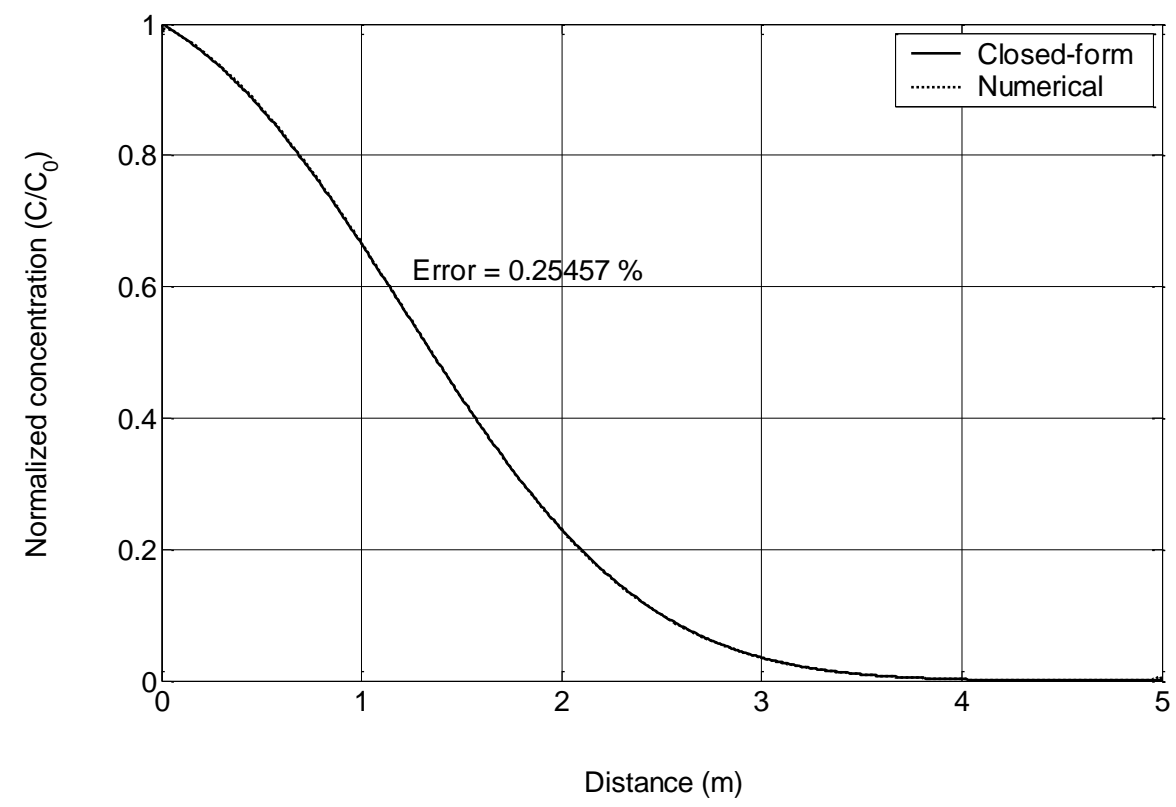

Figure 2: Advection-dispersion-reaction results, analytical and numerical solutions 
The analytical and numerical results are so close to each other that two distinct curves may be difficult or impossible to see. The error is on the order of $0.25 \%$, which is considered to be acceptable.

\section{Implementation: Modeling a Real-Case Scenario}

A computer simulation is an attempt to model a real-life or hypothetical situation. It is a tool to virtually investigate the behavior of a system under study by changing variables and initial or boundary conditions. All students were familiar with computer programming after taking $\mathrm{C}++$ course in their sophomore year, and were introduced earlier to Matlab scripts for the comparison of numerical and analytical solutions to the governing equations of contaminant transport. They were tasked to employ Matlab to simulate the concentration vs. time and concentration vs. distance of TCE using the data collected by the Environment Protection Agency (EPA) in the 2010 Annual Status Report for the Boomsnub/Airco Superfund Site, Hazel Dell, in Washington. The Boomsnub/Airco Superfund Site is located north of Vancouver in Hazel Dell, Washington. The site is a mix of residence, commercial and industrial properties and the TCE plume extends 4000 feet. There were already a number of different materials available online for learning the basics of Matlab, the most notable online material is the 'Introduction to MATLAB' course offered by Massachusetts Institute of Technology (MIT) through their OpenCourseWare program. The instructor also provided a list of basic functions for Matlab and examples of how to use them. For simplification purposes, students assumed that the concentration of TCE follows a first order degradation rate as a function of time and as a function of distance, when modeling the data. 


\section{Concentration vs. Time}

From the EPA website, students picked a well that was repeatedly sampled over time and plotted the TCE concentration vs. time for this location. AWM-16 well was sampled over 13 years and TCE concentration went down over time from $80 \mu \mathrm{g} / \mathrm{L}$ to less than $5 \mu \mathrm{g} / \mathrm{L}$. The EPA has set a maximum contaminant level (MCL) of $5 \mu \mathrm{g} / \mathrm{L}$ of TCE in drinking water. Figure 3 shows the site data and the Matlab modeling of the TCE concentration vs. time of AWM-16 well.

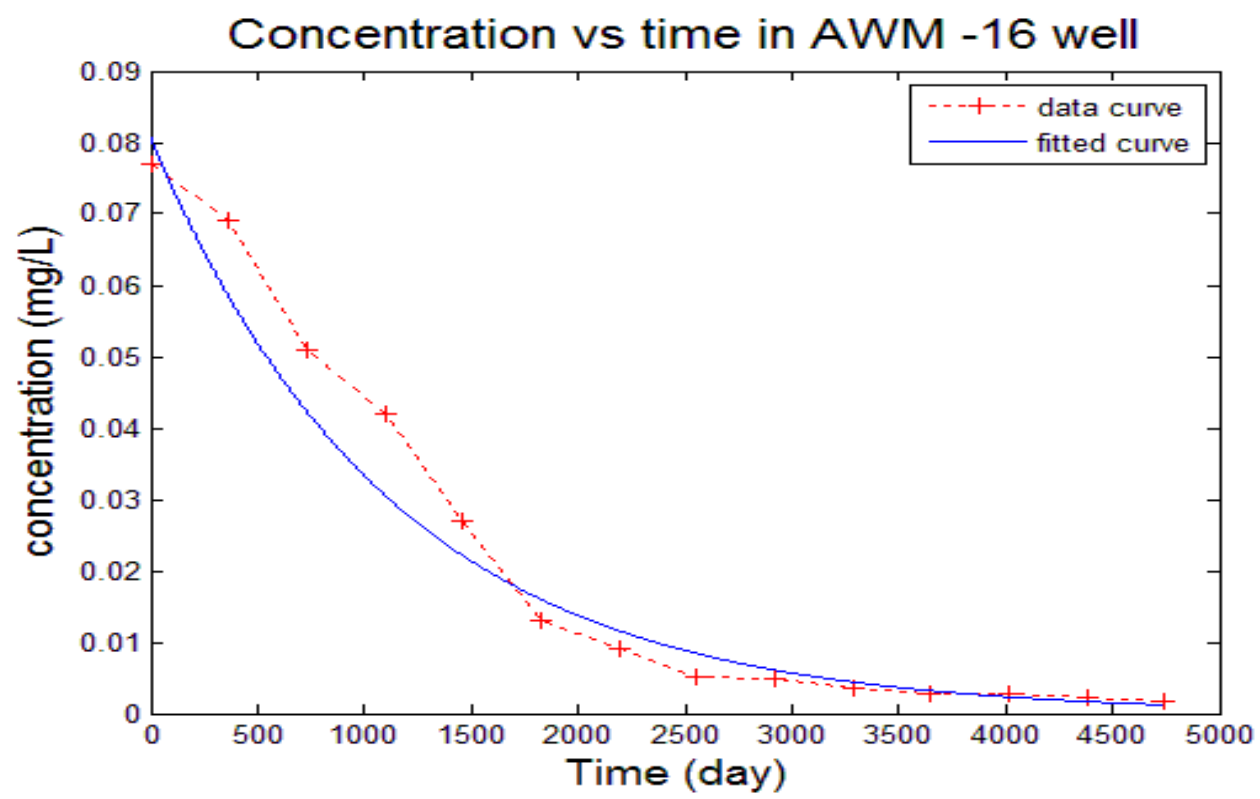

Figure 3: TCE concentration vs time of the data and fitted curve in AWM-16 well

\section{Concentration vs. Distance}

A one dimensional equation was employed to simulate the transport of contaminant over distance. Figure 4 shows the site data and the Matlab modeling of the TCE concentration vs. 
distance for the year 1997. For that year, even at 900 meters away from the source, the plume had a concentration of $100 \mu \mathrm{g} / \mathrm{L}$, twenty times more than the maximum allowed TCE level for drinking water.

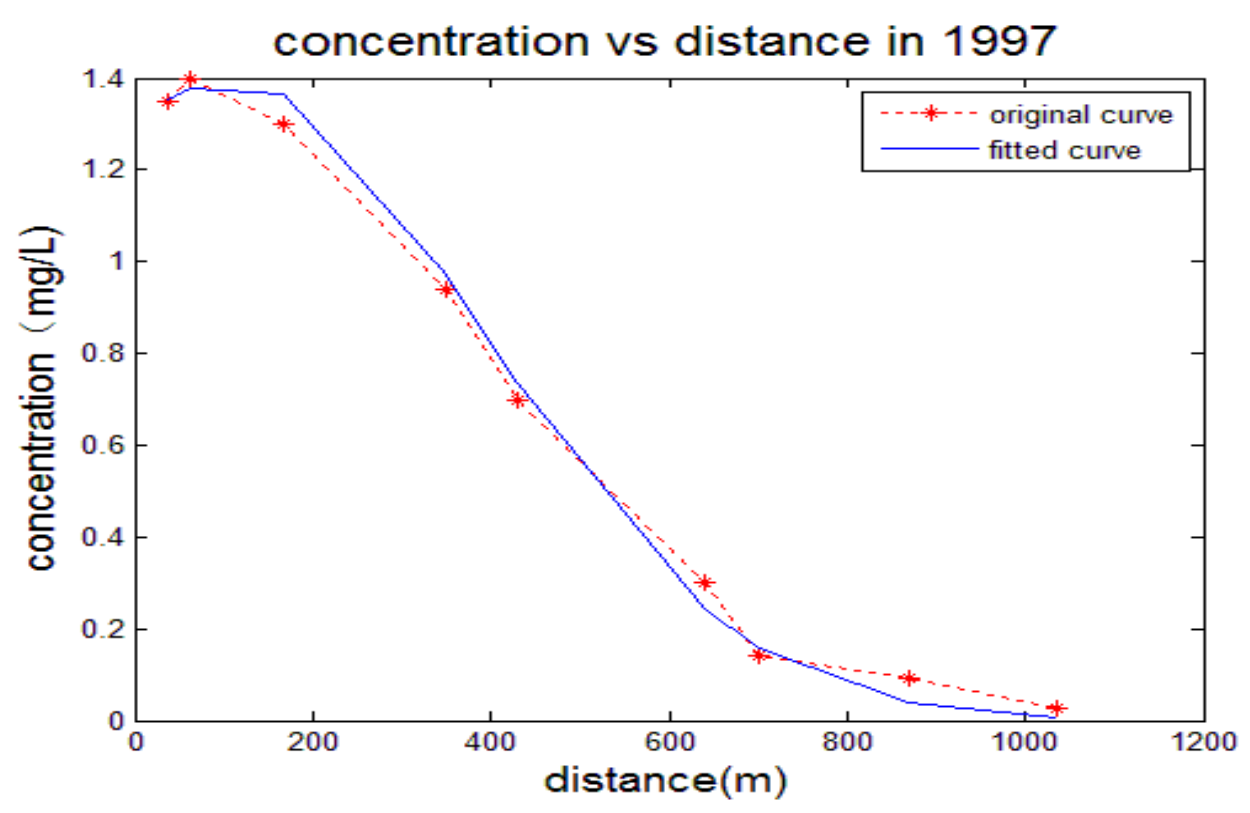

Figure 4: TCE concentration vs distance of the data and fitted curve in 1997

\section{Course Evaluation and Assessment}

Hazardous Waste course is two 75-minute lectures per week. With thirty-two students, eight groups of four were formed. Students had four weeks to complete the project. At the beginning of the project, one lecture was allocated to intoducing the students to Matlab resources. A list of basic Matlab functions and examples of codes were also provided. At the end of the four weeks, about a third of the class (35\%) did not finish the project on time and were given extensions. Several groups came asking for help throughout the duration of the project, even outside the instructor's office hours. 
At the end of the project, students were asked to answer a survey about their experience using Matlab. 47\% of the students stated that it was difficult to learn and write a MATLAB code, even though they have taken a $\mathrm{C}++$ course two years ago. Almost $85 \%$ of the students felt that more time was needed to complete the project. Some commented on the large amount of time spent watching online instructions of Matlab that were not necessarily needed for the project. However, when asked about the importance of learning a computer language, $92 \%$ stated that this skill and knowledge are very important and will help them in their job applications. They realize that employers are increasingly requiring from the engineering graduates an ability to use computer tools such as Matlab. When asked if the project helped with environmental concepts, $76 \%$ stated that modeling the plume helped them see the path and the time needed for the contamination to decrease. Similarly, course evaluations and comments at the end of the semester indicate that the Matlab modeling exercise, although challenging, was well received. Students were able to visualize when working on the project the spread of the plume and the concentration change with time. They were able to better understand and visualize the fate and transport of a contaminant in groundwater. Students were able to predict the time when the concentration of the contaminant drops below the maximum allowable concentration in drinking water and meet the EPA requirements. They also were able to model the movement of the plume over distance and predict when remedial actions become necessary to contain the spread of the plume. A discussion of policies and socio-economic considerations was held at the end of the project and the rationale behind the decision-making process in remediation techniques was examined. 


\section{Future Work}

Based on student feedback, class time would be allocated every week for students to work on their project. For future offerings, more time would be provided for the completion of the project. In addition, Matlab would be introduced earlier in the semester to allow for students to get familiarized with the code sooner. Group work presents its challenges too. Although, team work develops stronger communication skills, promotes responsibilities, and allows the instructor to assign more challenging projects, it is sometimes difficult to assess the individual contribution within the group and significant differences in concept learning and assimilation among the team members can be hidden. Thus for next offerings, group size will be decreased to two. Future work also includes technical and pedagogical development of the assignment. The technical development includes collecting data on more sites and adding features such as timevarying boundary conditions, multiple sources, and sinks. The pedagogical development includes assessing the student understanding of contaminant transport and fate prior to completing the computer assignment and after completing the computer assignment. 


\section{References}

Bear, Jacob. 1979. Hydraulics of Groundwater. New York, McGraw-Hill.

Burden, R. L. and Faires, J. D. 2001. Numerical Analysis $7^{\text {th }}$ Edition. Pacific Grove, CA, Brooks/Cole.

Cretchley, P., Harman, C., Ellerton, N., Fogarty, G. 1999. “Computation, Exploration, Visualisation: Reaction to MATLAB in First-year Mathematics," Proceedings of the Delta '00 Symposium on Undergraduate Mathematics, Toowoowba.

Dunne, B. E., Blauch, A. J., Sterian, A., “The Case for Computer Programming Instruction for all Engineering Discliplines," ASEE Annual Conference and Exposition, Conference Proceedings, 2005, pp. 1525-1537. Portland, OR.

Environmental Protection Agency:

https://www3.epa.gov/region10/pdf/sites/boomsnub_airco/2010annual_status_report_boo msnub_airco.pdf

Maase, Eric. 2007. "Kangaroo Thinking: Mathematics, Modeling, and Engineering in Introductory Computer Programming for Engineers," ASEE Annual Conference and Exposition, Conference Proceedings, 2007, Honolulu, HI.

Ogata, A., and Banks, R. B. 1961. A Solution of the Differential Equation of Longitudinal Dispersion in Porous Media. U.S. Geological Survey Professional Paper 411-A. 